Document downloaded from:

http://hdl.handle.net/10251/88300

This paper must be cited as:

Salvador Herranz, GM.; Contero, M.; Camba, J. (2014). Use of tangible marks with optical frame interactive surfaces in collaborative design scenarios based on blended spaces. Lecture Notes in Computer Science. 8683:253-260. doi:10.1007/978-3-319-10831-5_37.

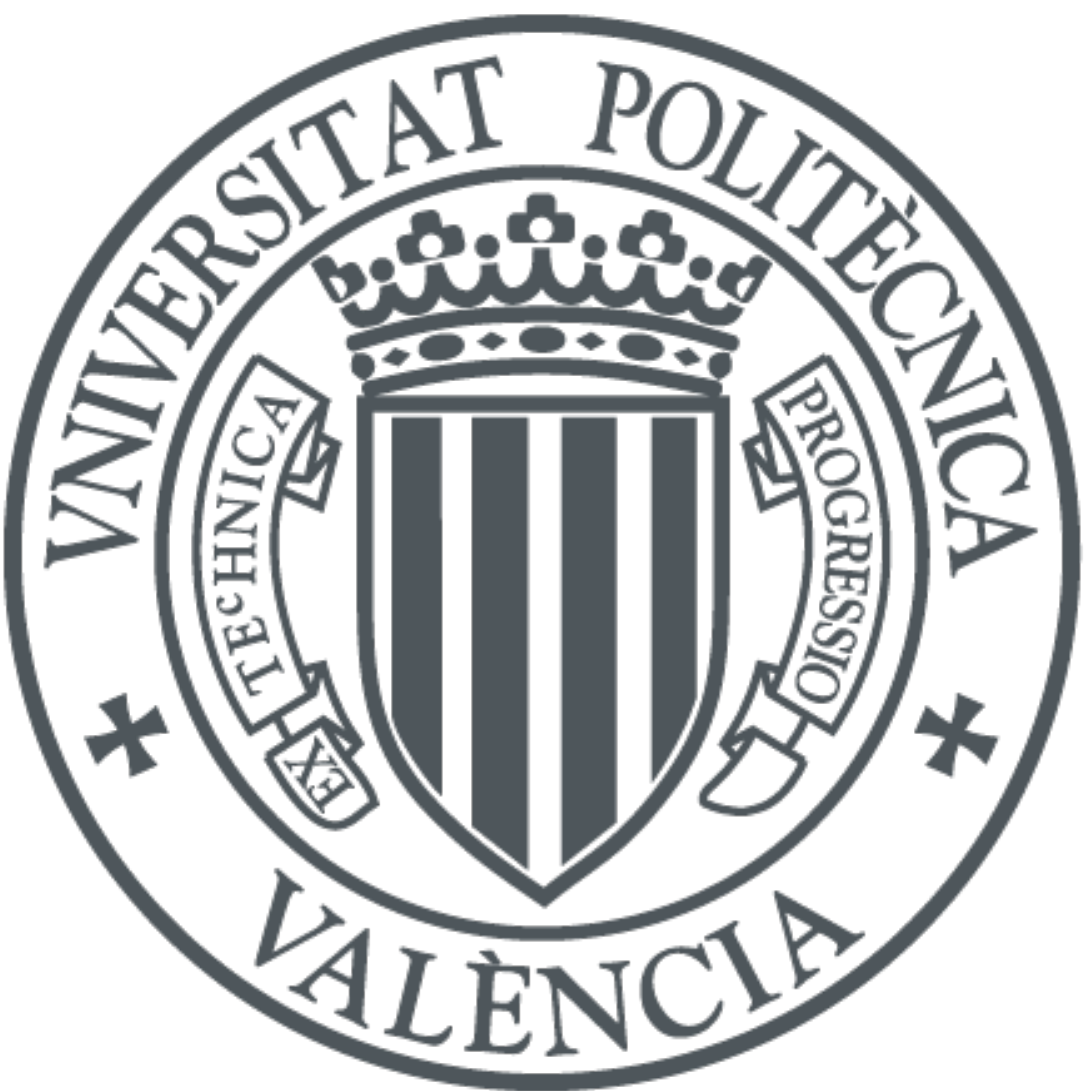

The final publication is available at

Copyright Springer Verlag (Germany)

Additional Information 


\title{
Use of Tangible Marks with Optical Frame Interactive Surfaces in Collaborative Design Scenarios Based on Blended Spaces
}

\author{
Gustavo Salvador-Herranz ${ }^{1}$, Manuel Contero ${ }^{2}$, Jorge Camba ${ }^{3}$ \\ ${ }^{1}$ Dpto. de Expresión Gráfica, Proyectos y Urbanismo, Universidad CEU Cardenal Herrera, \\ Valencia, Spain \\ gsalva@uch.ceu.es \\ ${ }^{2} \mathrm{I} 3 \mathrm{BH}$, Universitat Politécnica de Valencia, Valencia, Spain. \\ mcontero@upv.es \\ ${ }^{3}$ Engineering Design Graphics, Texas A\&M University, College Station, TX, USA \\ camba@tamu.edu
}

\begin{abstract}
In this paper, we present new methods of interaction with multi-touch surfaces implemented with optical frames by using tangible elements. Since interactive surfaces typically allow simultaneous detection of a large number of touch points, we propose the use of physical objects (which can be 3D printed) to reproduce different touch patterns, similar to using finger gestures on the table. These patterns are identified and recognized by our system and used to calculate the position and orientation of the mark on the surface. We propose the use of these tangible elements as a method to manage information locally in an interactive surface and also to exchange information between different surfaces to support collaborative design work.
\end{abstract}

Keywords. human-computer interaction, natural interfaces, blended spaces, interactive surfaces, tabletops.

\section{Introduction}

Teamwork is an essential element in engineering and industrial design activities, particularly during the early stages of the design process, where creativity plays a major role. In fact, many creativity techniques only make sense within multidisciplinary work groups. In these early stages, effective communication and exchange of ideas are critical. A major concern during the initial working sessions is to avoid introducing barriers that may hinder the communication process.

Although technology certainly improves the exchange of information among team members, it can also become a barrier during the early creative stages where communication must be direct and personal. Face-to-face meetings where designers sit around a table to discuss or generate new ideas (typically using some creativity technique such as brainstorming) are common. In this context, systems based on Natural Interfaces can make technology transparent to users so it does not interfere with the 
creative process. Similarly, Blended Spaces simplify the synthesis between analog and digital worlds, allowing users to move and manage information from one space to another in a natural and intuitive way.

Large multi-touch interactive surfaces are suitable systems for collaborative work. Such devices offer a large interactive surface that simulates a traditional work table. This environment facilitates face-to-face communication and promotes discussion and exchange of ideas. To be effective, these interactive surfaces must be multi-touch and multi-user, allowing multiple users to interact with the system simultaneously.

Interactive surfaces are particularly attractive when used as part of Blended Interactive Spaces, which are ubiquitous computing environments for computer-supported collaboration that builds on and enhances the pre-existing motor, spatial, social, and cognitive skills of groups of users [1-4]. Such spaces hide the complexity of technology and enhance Human Computer Interaction (HCI), making it more natural. Tangible User Interfaces play an important role in Blended Spaces, as they allow a type of human-machine interaction that is similar to natural processes.

Interactive surfaces have been implemented with different types of technology [5]. In this paper, we focus on the use of multi-touch systems constructed from optical marks. Such frameworks can transform any type of display device (projective or not) into an interactive multi-touch surface. In particular, flat-panel TVs with optical frames have been widely used as they can be turned into powerful systems with excellent image quality at a reasonable cost.

We present new forms of interaction with interactive multi-touch surfaces implemented with optical frames by using tangible elements, which are elements not originally designed for these devices. Since interactive surfaces typically allow simultaneous detection of $32+$ touch points, we propose the use of physical objects (which can 3D printed) to reproduce different touch patterns, similar to using finger gestures in certain positions on the table. These patterns can be identified and recognized by the system, providing its position and orientation on the interactive table.

\section{3D Tangible Marks}

Tangible user interfaces have been widely used as mechanisms to interact with digital information in a physical manner and a number of authors have demonstrated their benefits $[6,7]$. A variety of items can be used depending on the implementation of the interactive surface.

In general, for every tangible item in the system, it is necessary to maintain a unique identifier (ID) and track its position and orientation on the interactive table. For example, in rear projection based interactive surfaces (tabletops) a camera located below the surface recognizes predefined marks printed on the bottom faces of the tangible elements $[8,9]$, and calculates its position and orientation. Other implementations use radio frequency (RF) tracking methods. In this case, each tracked object contains an LC tag that resonates at a unique frequency. The position of the tag on the table is determined by measuring the resonance of the tag with different antenna elements on the tabletop. The problem with these systems is that only the XY position of 
the object on the surface (and in some cases an ID) is returned, but not the element orientation. Also, additional hardware is required, which increases the overall cost.

We propose the use of tangible elements in combination with interactive surfaces based on optical frames capable of detecting multiple touches (typically $30+$ ) simultaneously. Touch gestures in such systems are recognized when users' fingers interrupt the optical barrier created by the framework. However, there is no need to use fingers, as any object can be used to interrupt the optical beam. The main contribution of this study is the use of flat elements with pins located at the bottom that can interrupt the light beam generated by the optical frame. Digital information can be linked to and manipulated by these elements.

At least three pins are necessary (the vertices of a triangle) to always define a plane (see Fig. 1). The triangle defined by the three pins is isosceles so its position and orientation on the tabletop can be determined easily. As explained in the next section, the two long sides of the triangle are used to get the position and orientation of the mark on the tabletop. Additional pins placed between those that define the shortest side of the triangle are used to encode a unique ID of the mark in binary form. The higher the resolution of the optical frame, the more pins can be placed on the element and thus the more physical elements can be used. In the example shown in Fig. 2, three pins are used to encode eight different ID's. Fig. 3 shows another example in which 4 distinct marks are encoded, this time using just two pins. With this method, a total of $2^{n}$ unique IDs can be encoded, where $n$ is the maximum number of pins that can be placed in the shortest side of the triangle.
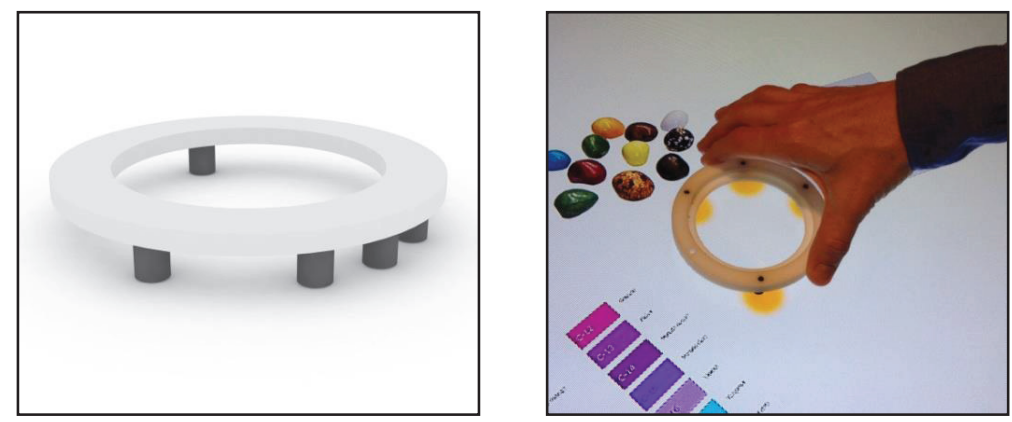

Fig. 1. Examples of Tangible Marks. Digital model (left) and real mark on tabletop (right)

\section{Information Retrieval from Tangible Marks}

To detect the location of the tangible marks on the interactive surface we use simple clustering techniques. The API of the optical frame provides a method to return the number and $\mathrm{XY}$ coordinates of each user action, which can be understood as a point cloud. To determine the clusters we use the Quality Threshold (QT) clustering algorithm. This is a simple method that does not provide the desired number of clusters (like in K-Means), but the maximum distance allowed for the cloud to be considered a cluster. In this case, the maximum distance is known (the diameter of the tan- 
gible mark). Although this algorithm is computationally intensive, the number of elements to classify is relatively small (about 32 , depending of the capabilities of the optical frame), so real-time classification is still possible.

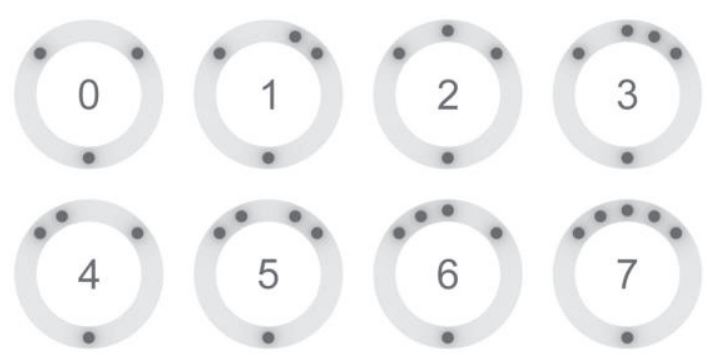

Fig. 2. Eight possible Tangible Marks using three encoding pins

Once the clusters are found, a convex hull for each of them is generated (see Fig. 3). Next, vectors $\overrightarrow{v 1 v 2}$ and $\overrightarrow{v 1 v 4}$ are defined using the two longest sides of the polygon, and added together to determine the direction vector of the mark. Additionally, the center $C$ of the mark can be found by transforming the direction vector into its unit vector and multiplying it by the radius of the mark.

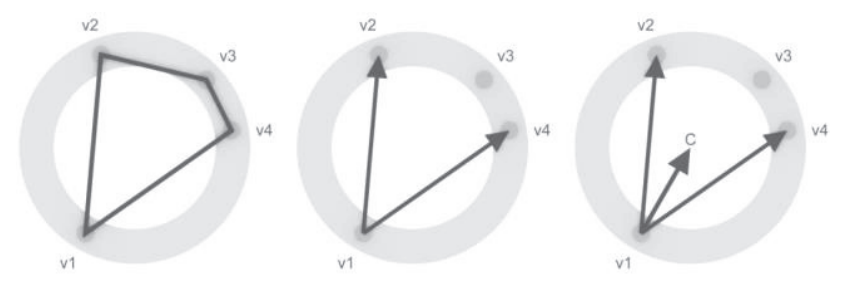

Fig. 3. Process for extracting position and orientation of a Tangible Mark: Convex hull (left). Main vectors (center). Position and orientation vector (right).

To get the ID of the mark, shape matching techniques can be applied [10]. We use shape matching by example using the algorithm described in [11]. This algorithm is not affected by rotation, scale and reference point, and also has a reasonable computational cost. The polygons that can be generated with two encoding pins are illustrated in Fig. 4.

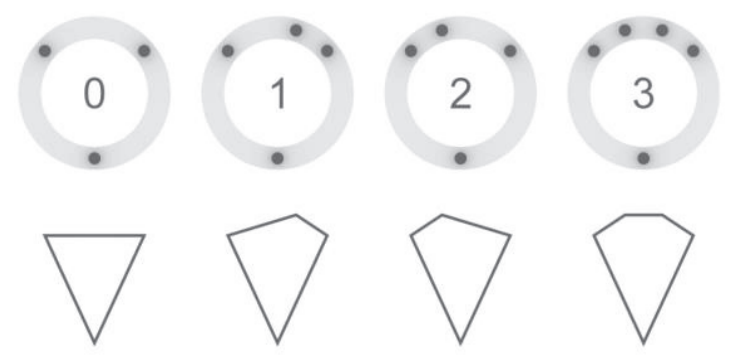

Fig. 4. Polygons associated with Tangible Marks 


\section{Distribute Collaborative Design Scenarios using Tangibles}

The combination of tangible marks with optical frame-based interactive surfaces represents a cost effective yet powerful mechanism to implement collaborative design scenarios based on Blended Spaces. In previous studies, [12] we proposed the use of interactive surfaces and designed a system based on natural interfaces that hides the complexity of technology and promotes face-to-face communication. Our system allows managing, organizing, and structuring digital two-dimensional graphical information and text in a natural way by simulating a physical workbench where designers traditionally work with paper, handwritten notes, sketches, pictures, and charts. This interactive surface can also be used to add information to the system in a variety of ways, such as by using shared folders in the cloud, via social networks like Twitter, or by sending messages with text and pictures.

A major feature of our system involves its distributed architecture, which allows teamwork in a co-localized manner. This means that different designers, or groups of designers (who may be geographically dispersed), can work collaboratively in real time using a common virtual board. The objective is to simulate a large common working plane (see Fig. 5) that can be shared by all users. An application server provides Internet support to the working plane and each device attached to the system acts as an interactive window to a section of this plane. These windows can be dragged and moved using simple finger gestures.

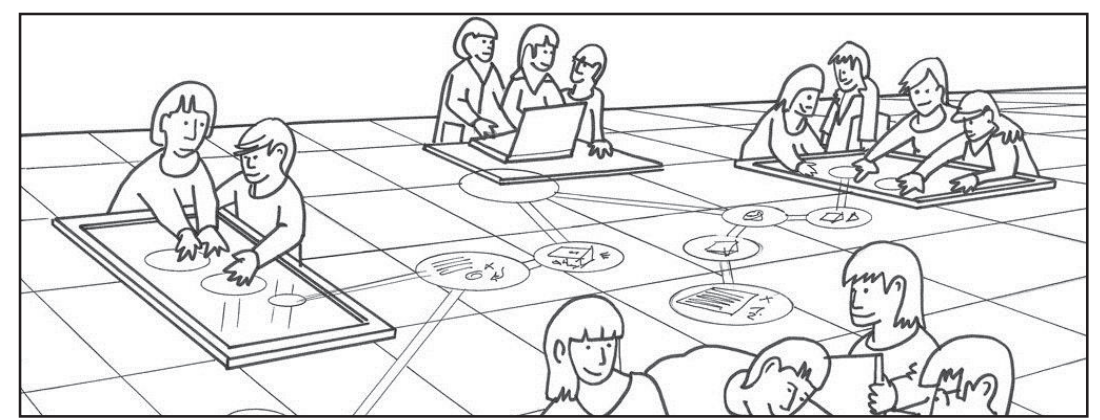

Fig. 5. Representation of the virtual work plane.

In this paper, we propose the use of tangibles as a method to manage information locally in an interactive surface and also to exchange information in a simple way between different interactive surfaces, thus extending the idea of blended spaces in distributed environments.

\subsection{Using Tangibles to Manage Information}

Our system can associate a tangible mark (using its identifier) to a data storage operation. When the mark is placed on the interactive surface, it is automatically recognized (as previously discussed) and data elements arranged on the tabletop can be 
linked to it simply by dragging them to touch the mark (the link is displayed graphically). If an object is already bound to the mark, repeating the same operation the object is unlinked. If the physical mark is removed from the table, all linked items disappear but remain stored in the application server (still associated with the identifier of the mark). Therefore, when the mark is placed again on the table, all elements reappear. If the mark is moved or rotated, all linked elements follow it hierarchically.

Similarly, it is possible to associate a mark with a deletion operation. In this functioning mode, when the mark is placed on the table objects can still be linked to it, but when removed, linked objects are removed from the application server. Therefore, if the mark is placed back over the table, elements will not reappear.

\subsection{Using Tangibles to Exchange Information Between Different Interactive Surfaces}

Tangible marks can also be used to exchange information in a simple and natural way between different interactive surfaces connected to the same application server. In addition, we can associate a mark with a "clipboard" so it can be used to perform copy-and paste operations, similar to the managing operations explained in the previous section, but without removing the information items from the interactive surface of origin.

\section{System Architecture}

Using the API functionalities provided by the optical frame manufacturer, we implemented a daemon process that continually samples touch gestures on the interactive surface and returns them as XY coordinates. With this information, the number of marks placed on the table as well as their respective IDs, position and orientation can be obtained, as described in section 3. At this point, using TUIO protocol [13], multiple TUIO "object" frames that correspond to those marks on the table and contain all their information (ID, position and orientation) are generated and sent to the system via UDP. All points that are not classified into any cluster (and thus do not belong to any mark) are sent as single points in single TUIO "point" frames. This feature allows compatible interaction with the multi-touch table using both tangible marks and hand gestures.

The application for collaborative work described in section 4 has been implemented using the Unity3D engine, which provides extensive support for developing graphical and networking applications (originally designed for the development of online games). Building on this engine, our system architecture is designed as a client-server application (see Fig. 6), where a server component supports the system, and multiple clients connect to it via TCP/IP. 


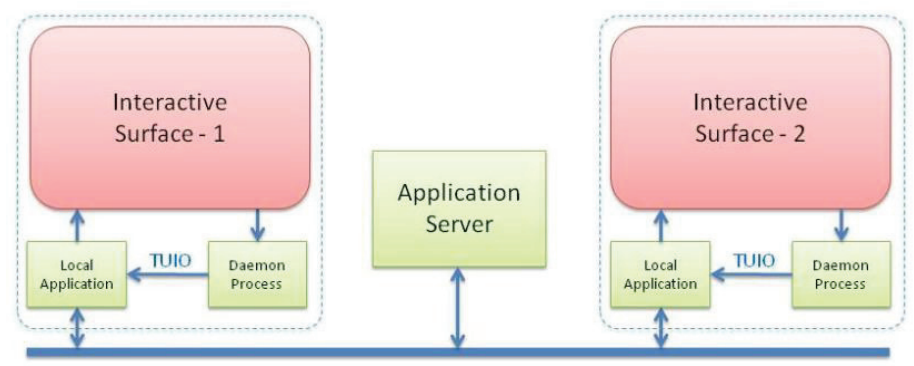

Fig. 6. Distributed System Architecture

The entire virtual workspace is managed by the server and each client has a view of it. Any action performed by a client is transmitted to the server, which immediately updates the scene and, in return, sends the changes back to the clients. The operation is similar to a network video game, where a common virtual world is stored on a server, and each player connects to it as a client. With this feature each interactive table has a view of a different area of a common space. For example, when a tangible mark is removed from a table, the associated items still exist in the application server (except in the case of delete operations) and are simply marked as hidden. When the mark placed again on the same or on another table, linked objects are moved to the new location and orientation, and re-marked as visible.

\section{Conclusions and Future work}

In this work, we have presented a new interaction mechanism based on tangible elements and interactive surfaces built with optical frames. The availability and gradually lower costs of large format flat screen TVs in conjunction with optical frames are providing affordable yet powerful tools to develop large interactive multi-touch systems with excellent image quality at reasonable prices.

With the incorporation of tangible items, interactive surfaces can be effectively integrated in environments based on Blended Spaces and Ubiquitous Computing. Although different methods to include tangible elements within these devices have been developed (such as those based on radio frequency or fiducial marks and overhead cameras), they all require additional hardware and occlusion problems may occur. The solution we propose uses existing hardware with no additional elements, which simplifies the system substantially. Additionally, our marks are passive and can be easily $3 \mathrm{D}$ printed. We have also integrated the TUIO protocol, which makes the system multiplatform and compatible with other development systems, thus simplifying the creation of new applications.

We are currently developing new tools and interaction mechanisms that use tangibles marks. Our goal is to facilitate teamwork and increase productivity by improving the ubiquitous aspects of the system. We are also interested in conducting a formal usability study of our tool to compare user performance and satisfaction when tangible marks are used. 


\section{$7 \quad$ References}

1. Benyon, D.: Presence in blended spaces. Interacting with Compututers, vol. 24 (4), 219226, (2012)

2. Hoshi, K., Öhberg, F., Nyberg, A.: Designing blended reality space: conceptual foundations and applications. Proceedings of the 25th BCS Conference on Human-Computer Interaction, 217-226, (2011)

3. Müller, D.: Mixed reality systems. International Journal of Emerging Technologies in Learning (iJET), vol. 5 (S2) (2009)

4. Benyon, D., Mival, O.: Blended spaces for collaborative creativity. Proceedings of Workshop on Designing Collaborative Interactive Spaces AVI2012 (2012.)

5. Müller-Tomfelde, C., editor: Tabletops - Horizontal Interactive Displays. Springer London. ISBN 978-1-84996-113-4. (2010)

6. Ishii, H., Ullmer, B.: Tangible bits: towards seamless interfaces between people, bits and atoms. Proceedings of the SIGCHI conference on Human factors in computing systems (CHI '97), 234-241. doi: 10.1145/258549 .258715 (1997)

7. Ishii, H.: Tangible user interfaces. The human-computer interaction handbook. Fundamentals, evolving technologies, and emerging applications. A. Sears \& J.A. Jacko (Eds.), 469-487. (2007)

8. Patten, J., Recht, B., Ishii, H.: Interaction Techniques for Musical Performance with Tabletop Tangible Interfaces. Advances in Computer Entertainment (2006)

9. Jordà, S., Geiger, G., Alonso, M., Kaltenbrunner, M.: The Reactable: exploring the synergy between live music performance and tabletop tangible interfaces. Proceedings of the 1st international conference on Tangible and embedded interaction. 139-146 (2007)

10. Veltkamp, R. C.: Shape matching: Similarity measures and algorithms. International Conference on Shape Modeling and Applications, SMI 2001. 188-197 (2001)

11. Arkin, E.M., Paul, L., Keden, K, Mitchell, J.: An Efficiently Computable Metric for Comparing Poygonal Shapes. Technical Report 89-1007. Department of Computer Science. Cornell University (1989)

12. Salvador, G., Bañó, M., Pérez, D., Contero, M.: A distributed collaborative learning tool based on a conceptual map paradigm and natural interfaces applied to the case of product design studies. Proceedings of the Research in Engineering Education Symposium (2003)

13. Kaltenbrunner, M., Bovermann, T., Bencina, R., Costanza, E,: TUIO - A protocol for table-top tangible user interfaces. Proceedings of the 6th International Workshop on Gesture in Human-Computer Interaction and Simulation (2005) 